\title{
Allograft Selection for Transepiphyseal Tumor Resection Around the Knee Using Three-Dimensional Surface Registration
}

\author{
Habib Bou Sleiman, ${ }^{1}$ Lucas E. Ritacco, ${ }^{2}$ Luis Aponte-Tinao, ${ }^{2}$ Domingo L. Muscolo, ${ }^{2}$ \\ Lutz-Peter Nolte, ${ }^{1}$ and Mauricio Reyes ${ }^{1}$ \\ ${ }^{1}$ Institute for Surgical Technology and Biomechanics, University of Bern, Stauffacherstrasse 78, 3014 Bern, Switzerland; \\ and ${ }^{2}$ Hospital Italiano de Buenos Aires, Gascón 450, Buenos Aires, Argentina
}

(Received 15 December 2010; accepted 16 February 2011; published online 1 March 2011)

Associate Editor James Tunnell oversaw the review of this article.

\begin{abstract}
Transepiphyseal tumor resection is a common surgical procedure in patients with malignant bone tumors. The aim of this study is to develop and validate a computerassisted method for selecting the most appropriate allograft from a cadaver bone bank. Fifty tibiae and femora were 3D reconstructed from computed tomography (CT) images. A transepiphyseal resection was applied to all of them in a virtual environment. A tool was developed and evaluated that compares each metaphyseal piece against all other bones in the data bank. This is done through a template matching process, where the template is extracted from the contralateral healthy bone of the same patient. The method was validated using surface distance metrics and statistical tests comparing it against manual methods. The developed algorithm was able to accurately detect the bone segment that best matches the patient's anatomy. The automatic method showed improvement over the manual counterpart. The proposed method also substantially reduced computation time when compared to state-of-the-art methods as well as the manual selection. Our findings suggest that the accuracy, robustness, and speed of the developed method are suitable for clinical trials and that it can be readily applied for preoperative allograft selection.
\end{abstract}

Keywords-Orthopedic oncology, Tumor resection, Allograft selection, Surface registration, Computer-assisted surgery.

\section{LIST OF SYMBOLS}

Variables

$\begin{array}{ll}b_{i} & i \text { th bone from the databank } \\ n & \text { Number of surface points of the search } \\ & \text { template }\end{array}$

Address correspondence to Habib Bou Sleiman, Institute for Surgical Technology and Biomechanics, University of Bern, Stauffacherstrasse 78, 3014 Bern, Switzerland. Electronic mail: habib.bousleiman@istb.unibe.ch

$\begin{array}{ll}p_{\mathrm{b}} & \text { Point on the bone from databank } \\ p_{\mathrm{s}} & \text { Point on the search template } \\ s & \text { Search template } \\ s_{\mathrm{T}} & \text { Transformed search template }\end{array}$

\section{ABBREVIATIONS}

cc Convergence criterion of ICP

CT Computed tomography

DB Databank

HSD Hausdorff surface distance (mm)

ICP Iterative closest point

maxIt Maximum number of iterations for ICP

MRI Magnetic resonance imaging

MSD Mean surface distance ( $\mathrm{mm}$ )

SD Standard deviation

T, TR Transformation matrix

\section{INTRODUCTION}

Transepiphyseal resection and consecutive reconstruction is a common surgical procedure in a wide number of patients suffering from malignant bone tumors. In this specific surgical scenario, the bone is cut in a way to preserve the epiphysis (Fig. 1d), and therefore an intercalary implant is required for the reconstruction. Various reconstruction methods exist, and the applicability of each of the methods is a strictly case-dependent decision. ${ }^{17}$ Biological reconstructions in great defects caused by transepiphyseal tumor resection around the knee is a major challenge of oncologic orthopedics. Clinical reports suggest that those defects can be repaired using bone allografts to 

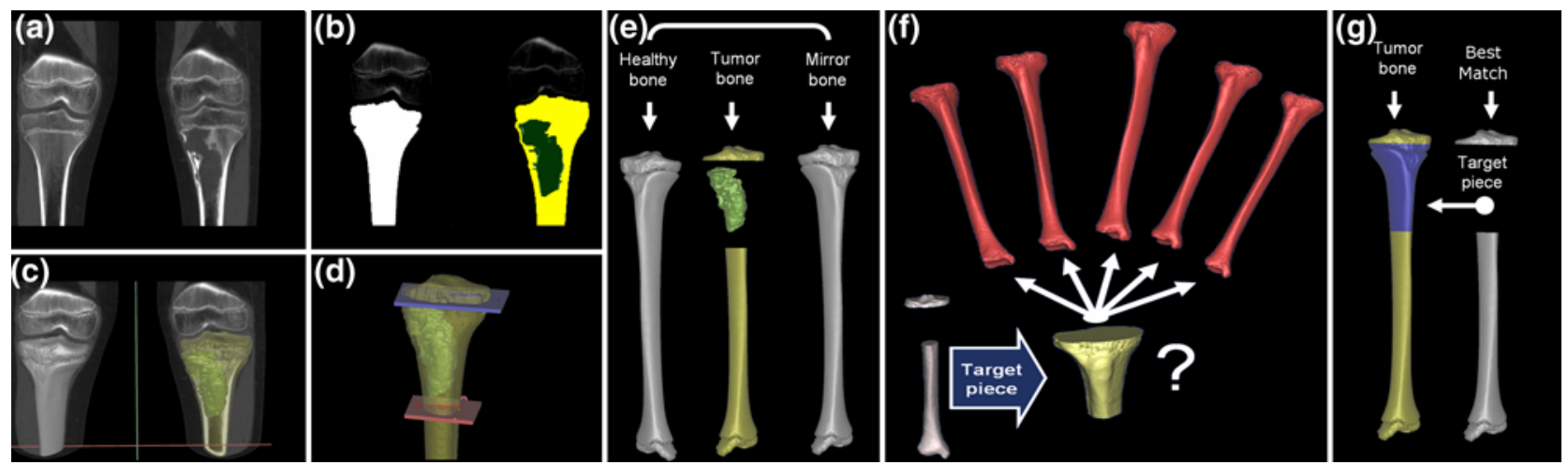

FIGURE 1. Processing pipeline. (a) Original CT image. (b) Segmentation mask. (c) 3D reconstruction of the tibiae and the tumor. (d) Cutting out the tumor in a virtual environment. (e) Illustration of the similarity between the diseased bone and the mirrored version of the contralateral tibia. (f) Illustration of how the template matching algorithm searches through the virtual bone database. (g) Illustration of the good fit of a part cut out from the best batching tibia and placed at the location of the resected section.

preserve the long-term bone stock and limb functionality. ${ }^{12,15-17,22}$ Matejovsky et al. ${ }^{12}$ and Ramseier et al. ${ }^{22}$ further support and promote the use of allografts as opposed to prosthetic intercalary implants, especially in younger patients. This was based on the long-term follow-up of their patients over time periods of several years. Moreover, Bielack et al., ${ }^{2}$ Matejovsky et al. ${ }^{12}$ and Paulussen et al. ${ }^{20}$ indicated that the tibia is a site presenting a high incidence of bone malignancies. A good allograft also facilitates and enhances the fitting process of the fixation plate(s). Furthermore, optimal handling of the bone bank ensures minimal loss of the usually scarce cadaver bone stock.

At present, we are not aware of any published automatic method able to select the best allograft around the knee from a virtual bone bank system based on shape analysis. However, recent studies demonstrated that a virtual model is a potential predictor to select the adequate allograft in a preoperative planning environment. ${ }^{19}$ Furthermore, shape matching is the chief method to be considered when a proper allograft is to be selected. ${ }^{6,7,18,19}$

This work is an extension of our previous work ${ }^{4}$ where we presented preliminary results on automatic allograft selection. However, the method presented several limitations. In particular, the degrees of freedom of the spatial search were only limited to the cranio-caudal direction, whereas no consideration to the remaining rotation and orientation parameters was regarded. Furthermore, the size of the validation dataset was reduced to ten patient computed tomography (CT) images, from which low resolution surface models of the femur were generated. Nevertheless, the obtained results were satisfactory, and brought forward a series of open-ended questions to be further investigated, relating to both the clinical and technical aspects of the method.
This study was also inspired by the findings of Ritacco et al., ${ }^{23}$ Schmidt et al., ${ }^{24}$ and Seiler et al.,${ }^{25}$ where it was shown that a pattern of symmetry between the contralateral lower limbs of the same subject does in fact exist. The search for the optimal allograft is automatically carried out through a databank of cadaver bones, in which the search is routinely performed manually. ${ }^{14,18,23}$ In a similar context, Paul et al. ${ }^{18,19}$ presented methods for the selection of massive bony allografts, in particular hemipelvic allografts. They employ either manual two-dimensional outline comparison or automatic three-dimensional image registration approaches to find the best donor. They also compare the two methods and show evidence that the registration method outperforms the planar outline matching. However, they consider the pelvis as a whole and do not discuss the problem of selecting an allograft for a localized region. They also do not simulate real clinical scenarios as they consider that the original anatomy of the pelvis is known beforehand. Towards the end of their discussion, they propose to rely on symmetry in order to recover information about the original anatomy of the patient at the tumor site.

This article presents a novel computer-assisted method for the selection of the allograft that best matches the patient-specific anatomy for transepiphyseal tumor resection around the knee. This work integrates concepts presented elsewhere, ${ }^{1,4,23-25}$ together with sound clinical aims and a more elaborate and comprehensive methodology, into an automatic system that provides the orthopedic surgeon with a relatively fast and accurate solution to the problem of selecting a good allograft. The functional outcome of the surgery is thus enhanced and the durability of the implant extended. ${ }^{12,15-17,22}$ The method adds to the recent research $^{19}$ the capability of selecting allografts for specific regions of the bone while taking into account 
that the presence of a tumor alters the original shape of the recipient bone.

This study aims to first assess the ability of the presented method to automatically select an adequately matching allograft. It also aims to explore whether it yields comparable or better results than those obtained by the manual method, and if its reproducibility is sufficiently reliable for clinical use.

\section{METHODS}

The method proposed in this article takes advantage of the aforementioned concept of symmetry to reconstruct the original shape of a diseased portion of the bone. A template corresponding to the location of the tumor is extracted from the patient's healthy contralateral bone. An iterative three-dimensional template matching process is then applied through the virtual cadaver bone databank to locate bone portions that resemble the template in terms of both morphology and scale. System testing and validation was carried out by simulating clinical cases from the available data.

The method presented herein was developed, tested, and validated using a set of 50 patient CT images of the lower limbs (varying image parameters and scanners). The bones were semi-automatically segmented using Amira ${ }^{\circledR}$ (Visage Imaging, Inc., San Diego, CA, USA), and stored in the form of surface point models and surface meshes (vertices per sample-tibiae: 42,004; femora: 58,837). This data is regarded as a digitally stored cadaver bone databank, in analogy to the one presented in Ritacco et al. ${ }^{23}$ From this point onward, we will be referring to those bones as cadaver bones.

The overall application of this method can be briefly described as follows: having a diseased bone, one can use the hereby presented tool in order to find amongst a set of healthy ipsilateral cadaver bones, the allograft that best matches the anatomy of the part to be resected. Knowledge about the original shape of that section is obtained from the contralateral bone of the same patient. This is achieved by first pre-registering the healthy contralateral bone to the diseased bone and manually cutting the part that corresponds to the location of the tumor. The processing pipeline therefore consists of the following steps, which are illustrated in Fig. 1:

(1) Acquisition of the CT images and segmentation of the patient's bones and tumor (Figs. 1a-1c)

(2) Virtually cutting out a part of the healthy contralateral bone that corresponds to the location of the tumor (Fig. 1d)
(3) Automatic registration of the template with all bones in the databank and storing measured distance metrics (Fig. 1f)

(4) Automatic selection of the closest (or few closest) match(es) from the databank (Fig. 1f)

(5) Using the boundaries of the registered template to outline the physical cutting planes on the selected bone and extract the allograft

As mentioned earlier, the original anatomy of the diseased bone is extracted from the patient's healthy contralateral limb and used as a template to guide the search within the databank of cadaver bones. This is illustrated in the form of a pseudo-code in Algorithm 1. For each cadaver bone in the databank (line 2), the algorithm applies an iterative closest point $^{1}$ (ICP)-based registration on the point clouds of the template and the bone itself to find the transform that minimizes the difference between the two surfaces (lines 4-7). This is done in an iterative fashion and only stops when a certain convergence criterion ( $\mathrm{cc}=0.001 \mathrm{~mm}$, line 8$)$ is met, or when the number of iterations exceeds a preset value (maxIt $=200$, line 8). Surface distance metrics are measured and stored for further processing (line 7). The rigid transformation is then applied to the template to place it in the best fitting location and orientation. This process is repeated until all bones in the databank are examined. An identity transformation is used to initialize the registration to avoid biased results. This could be on the expense of falling into local minima, but with the advantage of being able to find any matching bone segment along the potential donor bones and not only those close to the anatomical region of the tumor.

At this stage, each bone in the databank is represented by the minimum surface distance metric between the bone itself and the best fit of the template. Since the goal is to find the closest global match, one or more closely matching donors can be selected (lines 16, 17), thus giving the surgeon one-to-few possibilities to choose from.

\section{VALIDATION PROTOCOL}

\section{Simulated Clinical Cases}

A testing application was developed to assess the robustness of the proposed method and evaluate its possibility to be applied in a clinical setup. In every test iteration, the tool considers one dataset as a clinical case while using the remaining bones as the cadaver bones - the dataset taken as a clinical case is also included in the databank and used as a control sample, or what is referred to in Paul et al. ${ }^{18,19}$ as trap graft. 
ALGORITHM 1. Template matching algorithm.

\begin{tabular}{|c|c|}
\hline In: & $\begin{array}{l}\text { Search template } s \text { and all bones in the databank } \\
\mathrm{DB}=\left\{b_{1}, \ldots, b_{|| \mathrm{DB}||}\right\}\end{array}$ \\
\hline Out: & $\begin{array}{l}\text { Index of the closest matching bone in the databank } \\
\text { and the corresponding transformation parameters }\end{array}$ \\
\hline 1: & Initialize $j \leftarrow 0$; maxIt; cc \\
\hline 2: & for each $b_{i}$ in DB \\
\hline 3: & loop \\
\hline 4: & find corresponding points \\
\hline 5: & $\mathbf{T}_{j} \leftarrow$ estimate updated parameters \\
\hline 6: & $S_{\mathrm{T}} \leftarrow \mathbf{T}_{j} \circ S$ \\
\hline 7: & $d_{j} \leftarrow \operatorname{MSD}\left(s_{T}, b_{i}\right)$ \\
\hline 8: & if $\left(d_{j}-d_{j-1}\right)<\mathrm{cc} \| j>\max$ It then \\
\hline 9: & $D_{i} \leftarrow \min \left\{d_{k} \mid k=1 \ldots j\right\}$ \\
\hline 10: & $\mathbf{T R}_{i} \leftarrow \mathbf{T}_{\operatorname{argmin}}\left\{d_{k} \mid k=1 \ldots j\right\}$ \\
\hline 11: & break loop \\
\hline 12: & end if \\
\hline 13: & $j \leftarrow j+1$ \\
\hline 14: & end loop \\
\hline 15: & end for \\
\hline 16: & bestMatch $\leftarrow \operatorname{argmin}\left\{D_{i} i=1 \ldots\|\mathrm{DB}\|\right\}$ \\
\hline 17: & bestTransf $\leftarrow \mathbf{T R}_{\text {bestMatch }}$ \\
\hline
\end{tabular}

A clinical case consists of an assumingly diseased left bone, and its healthy contralateral counterpart belonging to the same patient. For every case, the template is cut out of the right-side bone and then fed to the template matching algorithm described earlier whose role is to find, within the bone databank, the bony part that best matches the shape of the template. The collective results of the validation experiments are listed in the following section.

Corresponding points between the template in its final position and the databank bone are efficiently computed by applying a space dividing $k$ d-tree data structure to the complete bone, and then selecting the closest points to those of the template. Surface distance metrics that were used for the validation of this method are the mean surface distance (MSD) and the Hausdorff surface distance (HSD). The former consists of the average value of the individual Euclidean distances between corresponding surface points. It provides information about the overall global similarity between the donor and the recipient. The latter is the largest amongst the individual Euclidean distances and it indicates the largest possible distance between the two surfaces. More formally, the distance metrics can be written as follows,

$$
\begin{gathered}
\operatorname{MSD}(\mathrm{s}, \mathrm{b})=\frac{1}{n} \sum_{k=1}^{n}\left\|p_{\mathrm{s} k}-p_{\mathrm{b} k}\right\|, \\
\operatorname{HSD}(\mathrm{s}, \mathrm{b})=\max \left\{\left\|p_{\mathrm{s} k}-p_{\mathrm{b} k}\right\| \mid k=1, \ldots, n\right\},
\end{gathered}
$$

where $k$ is the index of the search template point, $n$ the number of template surface points, and $\left\|p_{\mathrm{s} k}-p_{\mathrm{b} k}\right\|$ is the three-dimensional Euclidean distance between the $k$ th template point and its corresponding point on the surface of the examined bone. In Eqs. (1) and (2), s and $\mathrm{b}$ refer to the template and the cadaver bone, respectively.

\section{Performance Assessment}

A subset of ten clinical cases was used to evaluate the performance of the method relative to that of the manual approach. Two observers were asked to manually choose the best three matches for each template. Manual search was carried out using a computer interface with an interactive virtual environment within Mimics ${ }^{\circledR}$ (Materialise NV, Leuven, Belgium). Each result was individually scored. The Automatic method was applied in parallel and the best three matches for every template were noted and scored. The scoring system is based on a visual assessment of the fit of the allograft with particular attention to the overlap at the boundaries especially at the sites where a fixation plate would be placed.

Fisher's exact test was used to identify differences in the capability of both methods to detect the contralateral or trap graft. (The Chi-squared test was not chosen because the expected frequencies in the contingency tables were smaller than 5.) A significance level of 0.05 was chosen for all tests.

Agreement between both methods in choosing the three best matching allografts (with no consideration to their order) was assessed using Cohen's kappa. ${ }^{5}$ Using this result, it is possible to conclude about whether or not both methods are able to yield similar results.

Furthermore, Cohen's kappa was computed for the automatic method when applied four times on the same datasets. This measure would quantify the reproducibility of the method. Similarly, the reproducibility of the manual method was assessed by measuring the Cohen's kappa for the two observers.

\section{RESULTS}

In this section, the results of the validation protocol of the presented method are listed. The template matching algorithm was tested on a computer with a 32-bit architecture, $3.00 \mathrm{GHz}$ Intel ${ }^{\circledR}$ Core $^{\mathrm{TM}} 2$ Duo $\mathrm{CPU}$, and $3.25 \mathrm{~GB}$ of random access memory. The algorithm never failed to run or fell into numerical errors. Comparing a template to a single bone from the databank took $1.73 \pm 0.62 \mathrm{~s}$ (mean $\pm \mathrm{SD}$ ). These figures include the time to build the $k \mathrm{~d}$-tree whenever point correspondences are required. An overhead time of loading the database into memory and processing of 
the images is to be added, however, this can be done offline and is not different than that done in the manual method. Convergence of the iterative algorithm was mainly constrained by the preset convergence criterion. For the $50 \times 50$ comparisons, $97.24 \pm 27.96$ iterations were needed. Only three comparisons out of the total of 2,500 went over the limit of number of iterations.

In terms of surface distances, one would expect the best obtained match to be part of the contralateral bone of the same subject. This is due to the high similarity in the original morphology of the left and right sides of the patient. This was confirmed by the obtained results where an errorless classification was achieved. The control samples are therefore highlighted by the diagonals in Fig. 2, where the lowest MSD values were recorded. A similar diagonal pattern is demonstrated for the Hausdorff distance measurements.

The control samples can be considered as a further validation parameter, since clinically, the allograft must mimic the shape of the resected region as closely as possible, and therefore the template must match the shape of the missing part. The values occurring on the diagonals of the tables in Fig. 2 are $0.62 \pm 0.0066 \mathrm{~mm}$ (mean $\pm \mathrm{SD}$ ) in the case of MSD, whereas the HSD measurements are $2.30 \pm 0.76 \mathrm{~mm}$.

Figure 3 shows three-dimensional views of the results for the best match, the second best, and the worst for two different simulated clinical cases (i.e., two different patients). Surface distance is illustrated in the form of color-coded surface maps.

Tests comparing the performance of the proposed method to its manual counterpart were carried out on a subset of ten simulated clinical cases. Twenty manual (10 detections per observer) and 20 automatic detections were carried out in total. It took the observer on the average $12 \mathrm{~min}$ per template to manually search through the databank and give a score for each bone. The automatic method was able to correctly detect the symmetric template in all of the cases. The observers carrying out the process manually managed to correctly classify the trap graft in only 12 out of the 20 cases. Fisher's exact test proved an improvement of the automatic method over the manual approach $(p=0.002)$.

Cohen's kappa tests resulted in a value of $0.73(95 \%$ CI: 0.63-0.83), indicating an intraobserver agreement that is not accidental. ${ }^{11}$ The two methods were therefore yielding comparable results in terms of choosing the best three matches.

Reproducibility tests of the automatic method were carried out by applying the algorithm four times on the same datasets. In all cases, the algorithm converged to the exact same solution yielding a kappa value of 1.0 (95\% CI: $1.0-1.0)$. Reproducibility of the manual method, or agreement between the two observers, resulted in a kappa of 0.79 (95\% CI: 0.67-0.91).
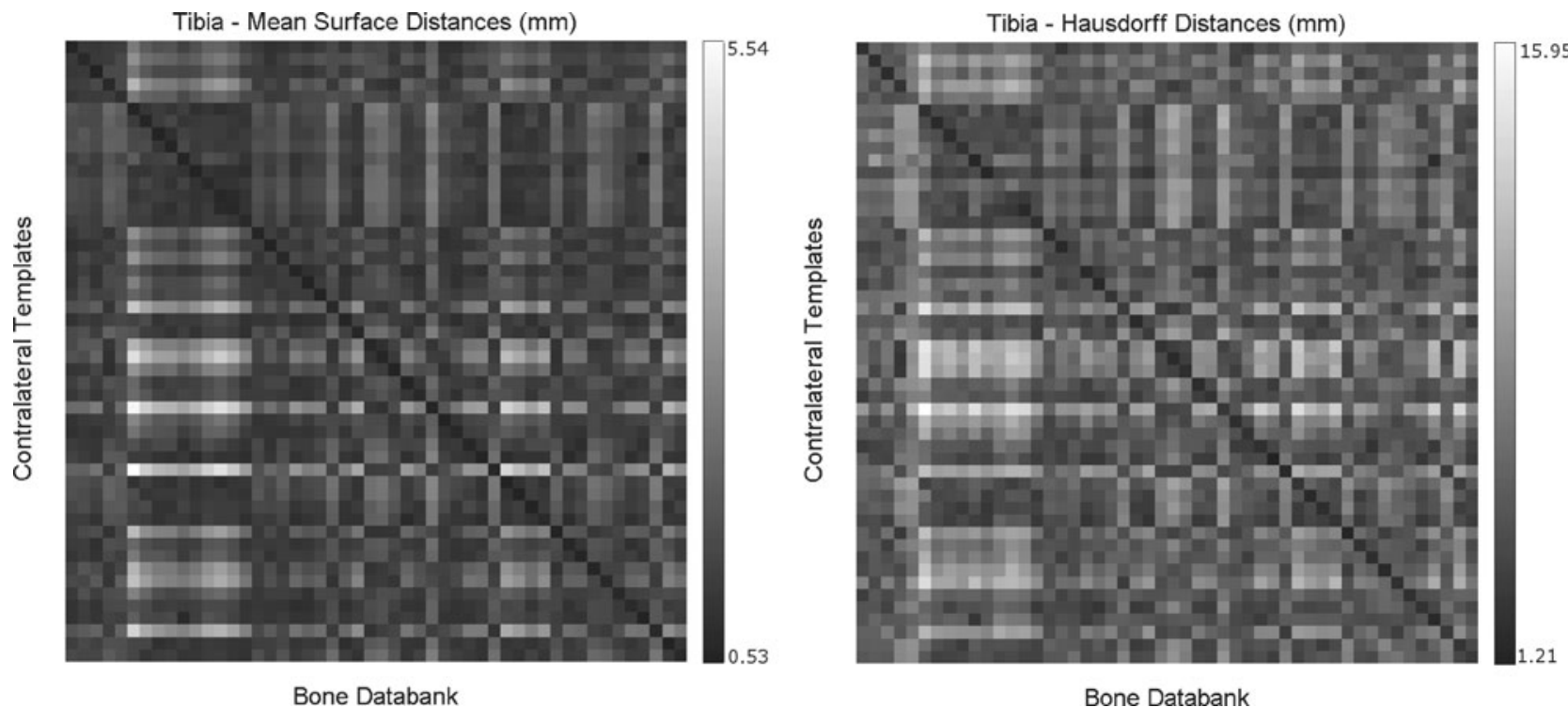

FIGURE 2. (Left) Mean surface distance (MSD) and (right) Hausdorff surface distance (HSD) grayscale-coded maps illustrating the results of the validation protocol. The vertical axis corresponds to the templates cut from the right tibiae, the horizontal axis corresponds to the left bones of the $\mathbf{5 0}$ different subjects, and the origin lies in the upper left corner. The low-intensity diagonals correspond to distances measured for the contralateral bones of the same patient. Each row corresponds to one simulated clinical case. 


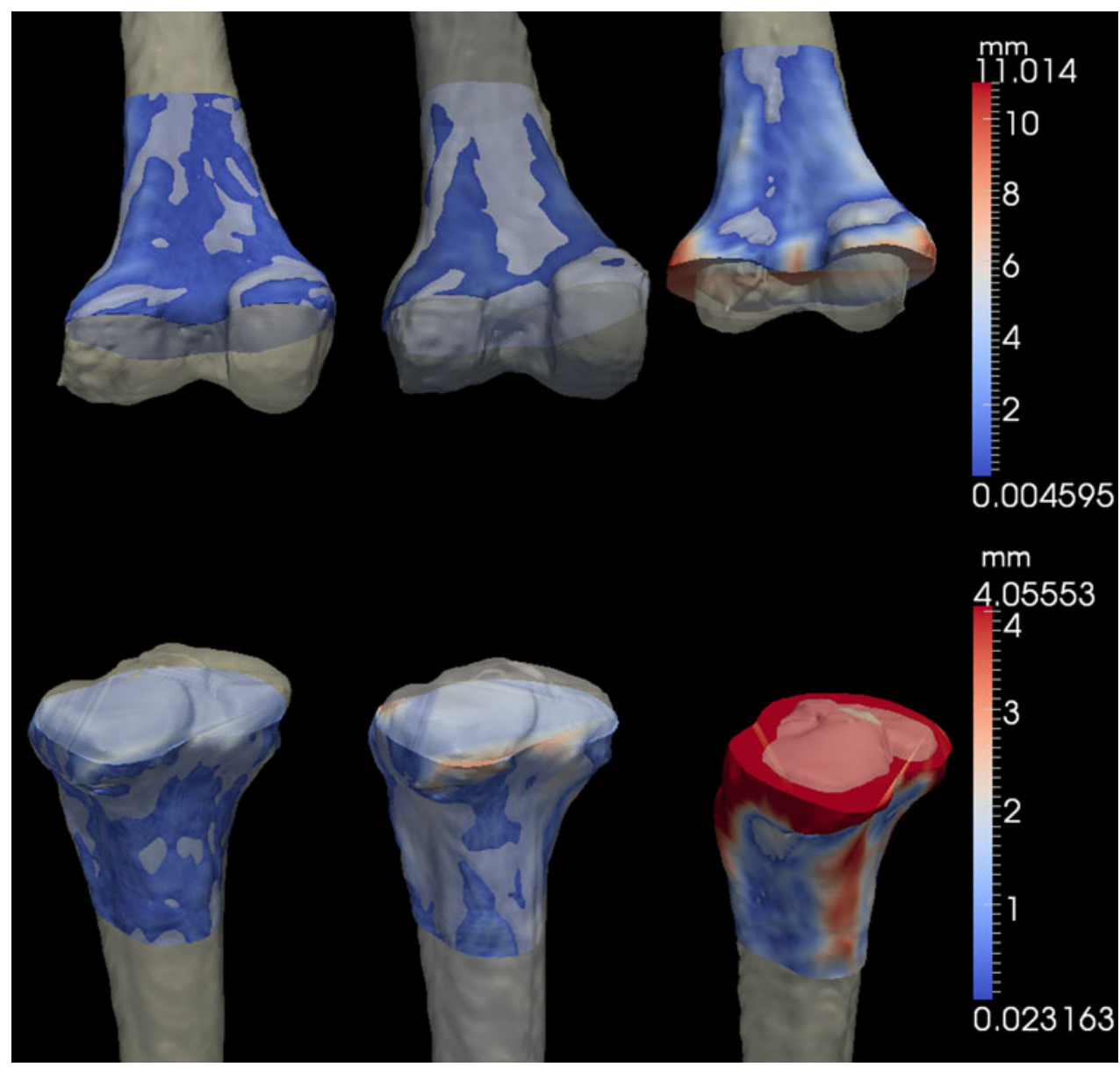

FIGURE 3. Three-dimensional view of a sample result. The surface distance between the template cut from the right bone and the left tibiae from the databank is represented as color-coded surface maps. The leftmost sample is the best match, the middle one is the second best, whereas the rightmost bone is the bone that presented the highest MSD.

\section{DISCUSSION}

In this article we presented a novel computer-aided method for the automatic selection of the donor allograft that best fits the patient-specific anatomy from a given virtual databank of cadaver bones. The method is able to speed-up and enhance the current state-ofthe-art that is employed in the clinical setup - a rather time-consuming and error-prone manual approach. A specific application was considered, namely, transepiphyseal tumor resection around the knee. Patientspecific anatomy is extracted from the healthy contralateral limb of the same individual. ${ }^{23-25}$

A thorough validation of the method was presented. Two distance metrics were presented and used in this article, in particular, MSD and HSD. The HSD metric, as well as the location of the region presenting the largest distance, are clinically relevant since they indicate whether or not the use of the particular allograft and the proper fitting of the fixation plate(s) is feasible.
Tests assessing the performance of the automatic method and comparing it to that of the manual method were as well carried out. The automatic method outperformed its manual counterpart in terms of detecting the contralateral bone, while maintaining a substantial agreement with the observers' choices of the best three matches. The presented method showed higher reproducibility than that measured for the experts.

The method can easily be extended to test different surgical scenarios such as epiphyseal, unicondylar, and bicondylar resections. Such scenarios might require intraarticular reconstructions where the cartilagineous tissue has to be considered. In such cases, and since CT does not provide adequate contrast in soft tissues, magnetic resonance images (MRI) can be used to complement the data acquisition system. Furthermore, and in order to circumvent the common shortage in donor bones, the search can be applied across dissimilar bones. For instance, a part of the femur can be grafted in the patient's tibia as long as it presents 
adequate morphology. We have plans to proceed with our research direction and further investigate those topics.

Computed tomography — and also MR — images are routinely used to determine the tumor resection margins. Given the current resolution of typical images, 3D segmentations and reconstructions usually offer highquality surface models that are adequate for further processing. Furthermore, the data used in the experiments of Bou Sleiman et al. ${ }^{4}$ was represented point models of a lower resolution (almost half the current resolution), and the results were nonetheless satisfactory. The results shown in the previous section support our initial hypothesis that an automatic three-dimensional mesh-based template matching algorithm could perform better than the state-of-the-art automatic and manual techniques. The presented computer-assisted method proved to be faster than the manual allograft selection and other reported approaches. ${ }^{18,19}$ When compared to the manual search, a significant improvement was recorded while maintaining a solid agreement between the expert's opinion and the outcome of the algorithm. Due to its mathematically stable nature, our method proved to be highly reproducible. Moreover, the algorithm did not fail to converge in any of the tested cases, and it yielded superior results to those obtained by the manual method. We therefore conclude that our method is based on reliable data and is accurate enough for clinical use.

The iterative closest point algorithm used in this work faces the inherent limitation of possibly falling into local minima, which could diverge the results from the sought solution. However, the results of our tests showed a good immunity of our method against these pitfalls. We are also aware that a good fit of the edges of the allograft is of higher importance than an overall good fit. We plan to investigate a weighted-ICP method in which the importance of the edges is emphasized by correspondingly weighting the vertices of the surface models.

Legal, ethical, and logistic issues are usually faced when a clinical technique requires the postmortem collection of organs. Nevertheless, our clinical partners have facilitated access to organ donation as the country they operate in has already adopted an opt-out presumed consent donation system. Seven other countries in Latin America share the same donation laws. ${ }^{13}$ Furthermore, bone banks collecting bones from donors who have agreed to sign an informed consent is currently part of the healthcare standards in several countries worldwide. ${ }^{6,7,9,10}$

In our initial assumption, we rely on symmetry between the right and left side of the patient to generate the search templates. Additional methods can also be investigated, for instance in the cases where there is a clear dissimilarity between the two limbs. Three-dimensional surface reconstruction and prediction methods, especially those based on a statistical shape model, ${ }^{3,8,21}$ are also capable of providing valuable information about the original shape of the operated bone. The large database of segmented long bones developed at our institute makes this kind of experiments feasible for future studies.

Our approach, however, searches for the best donor based on global surface characteristics. Nevertheless, displaying color-coded maps of local surface distances, as well as providing the surgeon with a choice of more than one candidate donor renders the tool more flexible and leaves the final decision to the expert.

\section{ACKNOWLEDGMENTS}

This work was carried out within the frame of the National Center of Competence in Research, Computer-Aided and Image-Guided Medical Interventions (NCCR Co-Me), supported by the funds of the Swiss National Science Foundation (SNSF).

\section{REFERENCES}

${ }^{1}$ Besl, P. J., and N. D. McKay. A method for registration of 3-D shapes. IEEE Trans. PAMI 14(2):239-256, 1992.

${ }^{2}$ Bielack, S., B. Kempf-Bielack, D. Schwenzer, T. Birkfellner, G. Delling, V. Ewerbeck, et al. Neoadjuvant therapy for localized osteosarcoma of extremities. Results from the cooperative osteosarcoma study group COSS of 925 patients. Klin. Padiatr. 211(4):260-270, 1999.

${ }^{3}$ Blanz, V., A. Mehl, T. Vetter, and H. P. Seidel. A statistical method for robust 3D surface reconstruction from sparse data. In: Proceedings of 2nd International Symposium on 3D Data Processing, Visualization, and Transmission, 2004, pp. 293-300.

${ }^{4}$ Bou Sleiman, H., L. Ritacco, and M. Reyes. Computerassisted allograft selection for transepiphyseal tumor resection at the knee. In: Proceedings of 10th Annual Meeting of CAOS International, 2010, pp. 91-94.

${ }^{5}$ Cohen, J. A coefficient of agreement for nominal scales. Educ. Psychol. Meas. 20(1):37-46, 1960.

${ }^{6}$ Delloye, C., X. Banse, B. Brichard, P. L. Docquier, and O. Cornu. Pelvic reconstruction with a structural pelvic allograft after re-section of a malignant bone tumor. J. Bone Joint Surg. (Am) 89A(3):579-587, 2007.

${ }^{7}$ Delloye, C., O. Cornu, V. Druez, and O. Barbier. Bone allografts-what they can offer and what they cannot. J. Bone Joint Surg. (Br) 89B(5):574-579, 2007.

${ }^{8}$ Fleute, M., and S. Lavallee. Building a complete surface model from sparse data using statistical shape models: application to computer assisted knee surgery. In: Proceedings of MICCAI 1998, pp. 879-887.

${ }^{9}$ Giannoudis, P. V., H. Dinopoulos, and E. Tsiridis. Bone substitutes: an update. Injury. 36(Suppl 3):S20-S27, 2005. 
${ }^{10}$ Hoeyer, K. An anthropological analysis of European Union (EU) health governance as biopolitics: the case of the EU tissues and cells directive. Soc. Sci. Med. 70(12): 1867-1873, 2010.

${ }^{11}$ Landis, J. R., and G. G. Koch. The measurement of observer agreement for categorical data. Biometrics 33(1): 159-174, 1977.

${ }^{12}$ Matejovsky, Jr., Z., Z. Matejovsky, and I. Kofranek. Massive allografts in tumour surgery. Int. Orthop. 30(6): 478-483, 2006.

${ }^{13}$ Mizraji, R., I. Alvarez, R. I. Palacios, C. Fajardo, C. Berrios, F. Morales, et al. Organ donation in Latin America. Transplant. Proc. 39(2):333-335, 2007.

${ }^{14}$ Mnaymneh, W., T. I. Malinin, J. T. Makley, and H. M. Dick. Massive osteoarticular allografts in the reconstruction of extremities following resection of tumors not requiring chemotherapy and radiation. Clin. Orthop. Relat. Res. 4(197):76-87, 1985.

${ }^{15}$ Muscolo, D. L., M. A. Ayerza, L. Aponte-Tinao, and G. Farfalli. Allograft reconstruction after sarcoma resection in children younger than 10 years old. Clin. Orthop. Relat. Res. 466(8):1856-1862, 2008.

${ }^{16}$ Muscolo, D. L., M. A. Ayerza, L. A. Aponte-Tinao, and M. Ranalletta. Partial epiphyseal preservation and intercalary allograft reconstruction in high-grade metaphyseal osteosarcoma of the knee. J. Bone Joint Surg. (Am) 86-A(12):2686-2693, 2004.

${ }^{17}$ Ozger, H., M. Bulbul, and L. Eralp. Complications of limb salvage surgery in childhood tumors and recommended solutions. Strategies Trauma Limb Reconstr. 5(1):11-15, 2010.

${ }^{18}$ Paul, L., P. L. Docquier, O. Cartiaux, O. Cornu, C. Delloye, and $\mathrm{X}$. Banse. Inaccuracy in selection of massive bone allograft using template comparison method. Cell Tissue Bank 9(2):83-90, 2008.
${ }^{19}$ Paul, L., P.-L. Docquier, O. Cartiaux, O. Cornu, C. Delloye, and X. Banse. Selection of massive bone allografts using shape-matching 3-dimensional registration. Acta Orthop. 81(2):252-257, 2010.

${ }^{20}$ Paulussen, M., S. Ahrens, J. Dunst, W. Winkelmann, G. U. Exner, R. Kotz, et al. Localized Ewing tumor of bone: final results of the cooperative Ewing's Sarcoma Study CESS 86. J. Clin. Oncol. 19(6):1818-1829, 2001.

${ }^{21}$ Rajamani, K. T., M. A. Styner, H. Talib, G. Y. Zheng, L. P. Nolte, and M. A. Gonzalez Ballester. Statistical deformable bone models for robust 3D surface extrapolation from sparse data. Med Image Anal. 11(2):99-109, 2007.

${ }^{22}$ Ramseier, L. E., T. I. Malinin, H. T. Temple, W. A. Mnaymneh, and G. U. Exner. Allograft reconstruction for bone sarcoma of the tibia in the growing child. $J$. Bone Joint Surg. ( Br) 88(1):95-99, 2006.

${ }^{23}$ Ritacco, L. E., A. E. Orias, L. A. Tinao, D. L. Muscolo, F. G. Bernaldo de Quirós, and I. Nozomu. Threedimensional morphometric analysis of the distal femur: a validity method for allograft selection using a virtual bone bank. In: Proceedings of 13th World Congress on Medical and Health Informatics, MedInfo, 2010, pp. 12871290.

${ }^{24}$ Schmidt, W., M. Reyes, F. Fischer, R. Geesink, L. P. Nolte, J. Racanelli, et al. Quantifying human knee anthropometric differences between ethnic groups and gender using shape analysis. In: Proceedings of Annual Meeting American Society of Biomechanics, 2009, pp. 26-29.

${ }^{25}$ Seiler, C., S. Weber, W. Schmidt, F. Fischer, N. Reimers, and M. Reyes. Automatic landmark propagation for left and right symmetry assessment of tibia and femur: a computational anatomy based approach. In: Proceedings of 9th Annual Meeting of CAOS International, 2009, pp. 195-198. 